\title{
FLOCCULATION OF FLOTATION TAILINGS USING THERMOSENSITIVE POLYMERS
}

\author{
Wojciech Bogacz $^{* 1}$, Marcin Lemanowicz ${ }^{1}$, Andrzej Gierczycki ${ }^{1}$, Anna Mielańczyk ${ }^{2}$ \\ ${ }^{1}$ Silesian University of Technology, Faculty of Chemistry, Department of Chemical Engineering and \\ Process Design, ul. ks. M. Strzody 7, 44-100 Gliwice, Poland \\ ${ }^{2}$ Silesian University of Technology, Faculty of Chemistry, Department of Physical Chemistry and \\ Technology of Polymers, ul. ks. M. Strzody 9, 44-100 Gliwice, Poland
}

The key feature of thermosensitive polymers is the reversible transition between the hydrophilic and hydrophopic forms depending on the temperature. Although the main research efforts are focused on their application in different kinds of drug delivery systems, this phenomenon also allows one to precisely control the stability of solid-liquid dispersions. In this paper research on the application of poly(N-isopropylacrylamide) copolymers in processing of minerals is presented. In the experiments tailings from flotation plant of one of the coal mines of Jastrzębska Spółka Węglowa S.A. (Poland) were used. A laser particle sizer Fritsch Analysette 22 was used in order to determine the Particle Size Distribution (PSD). It was proved that there are some substantial issues associated with the application of thermosensitive polymers in industrial practice which may exclude them from the common application. High salinity of suspension altered the value of Lower Critical Solution Temperature (LCST). Moreover, the co-polymers used in research proved to be efficient flocculating agents without any temperature rise. Finally, the dosage needed to achieve steric stabilization of suspension was greatly beyond economic justification.

Keywords: aggregation, flocculation, thermosensitive polymers, PNIPAM

\section{INTRODUCTION}

The key feature of thermosensitive polymers is the reversible transition between the hydrophilic and hydrophopic forms depending on the temperature (Cheng et al., 2006; Kuźnik et al., 2010; Sakohara et al., 2008; Xing et al., 2014). The phenomenon occurs after passing the Lower Critical Solution Temperature (LCST) as a result of shape change of polymer chains from extended coils into collapsed globules. Poly(N-isopropylacrylamide) (PNIPAM), as the most common example of thermosensitive polymer, is a non-toxic polymer which can be used as environmentally friendly substitute to industrial agents (Burdukova et al., 2010a; Ghimici and Constantin, 2011; Seidi and Heshmati, 2015; Yan et al., 2010). Moreover, Forbes (2011) indicated that PNIPAM may be more efficient than conventional flotation agents. Its copolymers can be used for selective removal of desired mineral (Burdukova et al., 2010b). At the present moment, one may find papers where thermosensitive polymers were used for suspension flocculation (Burdukova et al., 2011; Deng et al., 1996; Forbes, 2011; Ghimici and Constantin, 20011; Kuźnik et al., 2010; Lemanowicz et al., 2012; Mori et al., 2013; Sakohara et al., 2008; Sakohara et al., 2013), nanoparticles stabilization (Budgin et al., 2012; Gong et al., 2012) or heavy metal ion removal (Tokuyama et al., 2010). Franks proved that cooling of sediment leads to enhanced consolidation (Franks, 2005). He used zircon suspension and methylcellulose as the thermosensitive agent. Identical conclusions were made in other papers (Franks et al., 2009; Li et al., 
2009). Thus, research of new materials and phenomena associated with thermoresponsive polymers is of great interest in the areas of selective separation, process control, flotation, dewatering and generally speaking environmentally sustainable technology.

The behavior of thermosensitive polymers and associated particulate processes depend on many factors. The most important are: molecular weight, molecular structure, $\mathrm{pH}$ and temperature (Budgin et al., 2012; Burdukova et al., 2010; Burdukova et al., 2011; Deng et al. 1996; Liu et al., 2001; Qin et al., 2014; Yue et al., 2012, Zhang et al., 2013). Moreover, in the case of flocculation three additional factors come into play; polymer dose, addition method and heating method (Lemanowicz et al., 2012). Any change of these parameters may influence the aggregation mechanism significantly.

The aggregation via standard flocculation runs in four steps (Gregory, 1993). In the first step a polymer is added to the solution and they are mixed. In the second step polymer chains are adsorbed on the surface of particles and then, in the third step, they undergo reconformation. At this point the suspension is destabilized and particles may aggregate with each other as a result of following three mechanisms: charge neutralization, charge patching or bridging. In the first case the electrostatic charge between particles and solution is reduced which allows them to aggregate via Van der Waals forces. In the second case polymer chains adsorbed on particles create charge patches in the electrostatic barrier through which particles can connect with each other. Finally, in the third case polymer chains create bridges between solid particles. What is important in this process is irreversible (Šulc et al., 2012; Tadros, 2013).

In the case of thermosensitive aggregation, aggregates are formed due to hydrophobic interactions between particles (Lemanowicz et al., 2016a). However, the transformation of macromolecules as a result of temperature change is hampered due to their adsorption onto the surface of particles (Kuźnik et al., 2010). Generally, in this case the higher the temperature of suspension the more complete the polymer transition is. Particles covered with a hydrophobic polymer tend to aggregate in order to reduce their free energy and, as a result, they create large aggregates. When the suspension is cooled down the breakage phenomenon occurs because the surface of particles becomes hydrophilic (Burdukova et al., 2011; Kuźnik et al., 2010; Lemanowicz et al., 2016b; Sakohara et al., 2008).

So far, studies on thermoresponsive flocculants were mainly focused on model suspensions. However, additional factors have to be taken into consideration if such systems are to be used in practical applications. In the case of typical industrial slurries the thermoresponsive flocculation process can be expected to be affected by the presence of salt, acidity/alkalinity and possibly other substances. In the presented research the flocculation of suspension of industrial origin was investigated. The aim of this study was to verify the possibility of practical application of thermosensitive polymers, especially in the mineral processing technology. The results concerning flocculation of a coal mine flotation tailings with a thermoresponsive polymer based on poly( $\mathrm{N}$-isopropylacrylamide) are presented. The research was focused on PSDs and their change during destabilization of the system.

\section{EXPERIMENTAL}

Samples of flotation tailings were taken from one of the mines of Jastrzębska Spółka Węglowa S.A (Poland). In order to receive representative samples the suspension was collected during normal work of flotation plant into two $50 \mathrm{dm}^{3}$ barrels. In the industrial practice, in the next step, it would be mixed with a flocculant and transferred to a Dorr settler. 

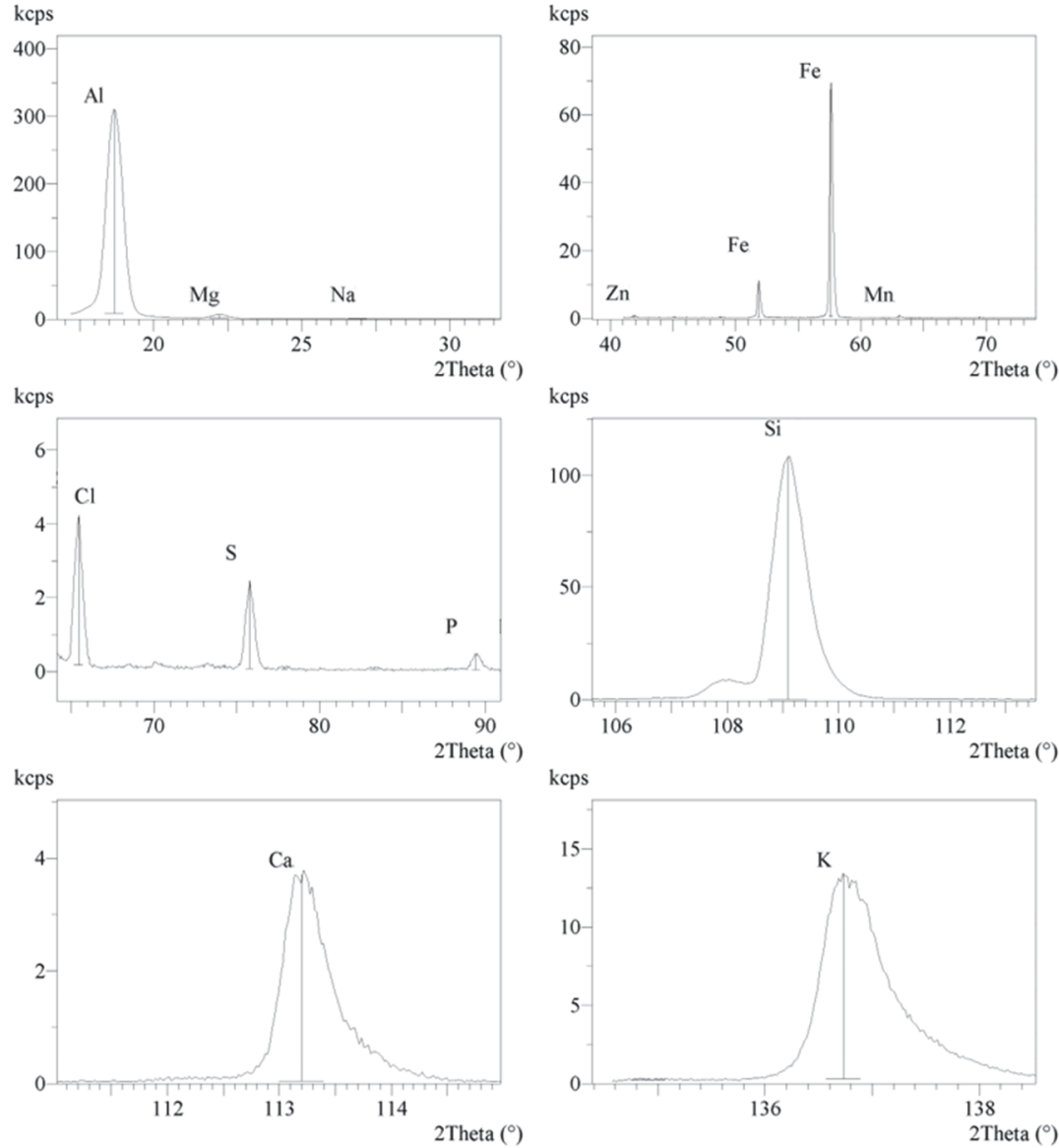

Fig. 1. Chemical composition of flotation tailing solid matter
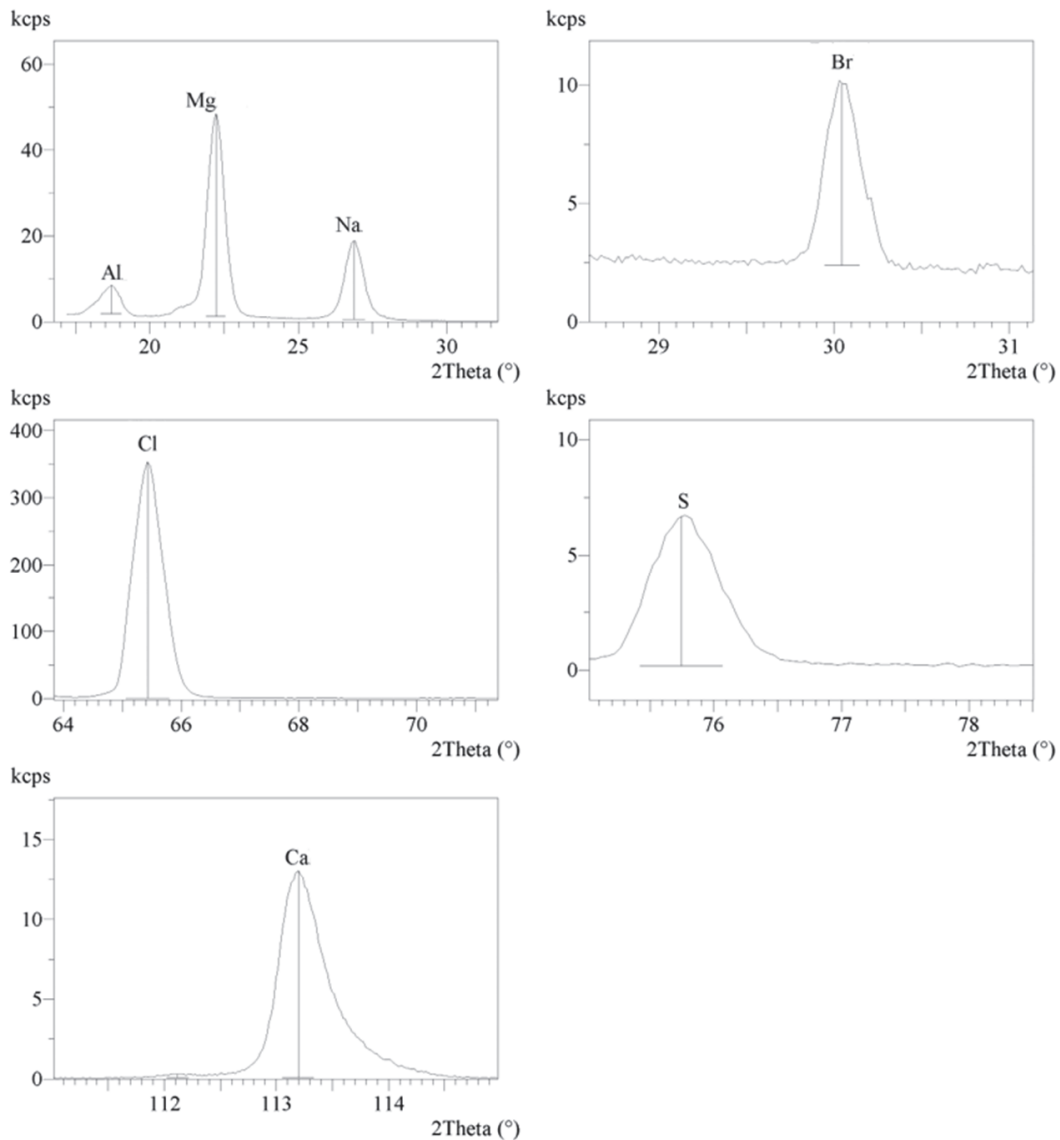

Fig. 2. Chemical composition of flotation tailing filtrate 
The samples had a form of diluted, gray suspensions. The chemical composition of the samples was determined using a Philips PW2400 spectrometer (Fig. 1 and 2). As expected, the solid matter was mainly composed of aluminum, silicon and iron compounds with some trace amounts of other elements. In the case of filtrate it contained dissolved salts of magnesium chloride, sodium chloride, calcium chloride and smaller amounts of other elements. The size of particles was determined based on the laser light scattering method using Fritsch Analysette 22. The minimum size of particles was equal to $0.15 \mu \mathrm{m}$ (measurement limit), the maximum size was equal to $40 \mu \mathrm{m}$ whereas the dominant value was equal to $5 \mu \mathrm{m}$. Finally, the Sauter mean diameter $\mathrm{d}_{32}$ was equal to $2.65 \mu \mathrm{m}$. The zeta potential of the suspension was measured using Malvern Nano $Z$ equipment. Due to the limitations of particle size that can be analyzed, a $100 \mathrm{ml}$ of suspension was taken and subjected to settling in a measuring cylinder. Next the fraction of the smallest particles which were present at the top of suspension was collected and their zeta potential was measured. It was equal to $(-20.1) \mathrm{mV}$. The mass of suspended solids as well as salinity were determined on the basis of the weight method. For that purpose a small portion of suspension was placed in a beaker and dried in a laboratory dryer at the temperature of $378 \mathrm{~K}$ as long as its mass was constant. Simultaneously, another sample of suspension was filtrated using the Büchner funnel. The filtrate was collected and evaporated in the laboratory dryer in the same conditions as the suspension. The desired parameters were calculated using difference in mass of the samples before and after drying/evaporating. Concluding, the mass fraction of suspended solids was equal to $1.57 \% \mathrm{w} / \mathrm{w}$ and the salinity was equal to $1.62 \% \mathrm{w} / \mathrm{w}$.

In the experiments two different polymers were used as the flocculants. The intentions of the authors were to synthesize two destabilization agents, one of cationic nature and one of anionic nature, with similar weight average molecular weight and transition temperature. Such samples would allow one to research the flocculation process in a qualitative way without interference on the physicochemical properties of industrial samples. Unfortunately, the incorporation of the ionic element into the polymeric chain alters the LCST significantly (Sakohara et al., 2008; Sakohara et al., 2013). Therefore considering economical aspects of the investigated process the molar ratio of ionic repeating units to nonionic ones had to be very low in order to keep LCST at a reasonable level. The synthesis procedure was identical to that presented in (Kuźnik et al., 2010). The only difference was the type and amount of the second comonomer. The polymer samples were synthesized in free radical polymerization with redox initiating system - ammonium persulfate (98\% purity, Acros Organics, Avantor Performance Materials), and tetramethylethylenediamine (TEMED) (99\% purity, Sigma-Aldrich) (Fig. 3). The comonomers were used as received: N-isopropylacrylamide (NIPAM, Acros Organics, Avantor Performance Materials) and methacrylic acid (MAA, Sigma Aldrich) or diallyldimethylammonium chloride (DADAMAC, Sigma-Aldrich) in 99:1 molar ratio (Fig. 4). The reaction mixture was purged with an inert gas for one hour before the addition of TEMED. Reaction products were separated by thermoprecipitation - the reaction mixture was heated to induce polymer separation from low molecular weight reagents and water. The thermoprecipitaiton was repeated twice, and the polymers were then dried to constant mass at $378.15 \mathrm{~K}$, weighed and dissolved in Reverse Osmosis (RO) purified water. The weight average molecular weights $\left(M_{w}\right)$ of the polymers were determined on the basis of the Static Light Scattering (SLS) measurements using Malvern Nano S90 applying the Rayleigh equation which describes the intensity of light scattered from a particle in a solution. The Debey plot for each polymer was obtained from several individual measurements: a zero concentration measurement (water) and samples of various polymer concentrations which allowed to determine $\mathrm{M}_{\mathrm{w}}$. In both cases the average weight molecular weights were comparable and were approximately $1200 \mathrm{kDa}$. Attempts have also been made to perform a molecular weight measurement using the size exclusion chromatography (SEC). Unfortunately, the high viscosity of polymer solutions, probably caused by relatively high $\mathrm{Mw}$, disabled the preparation of samples with appropriate concentration for analysis. 


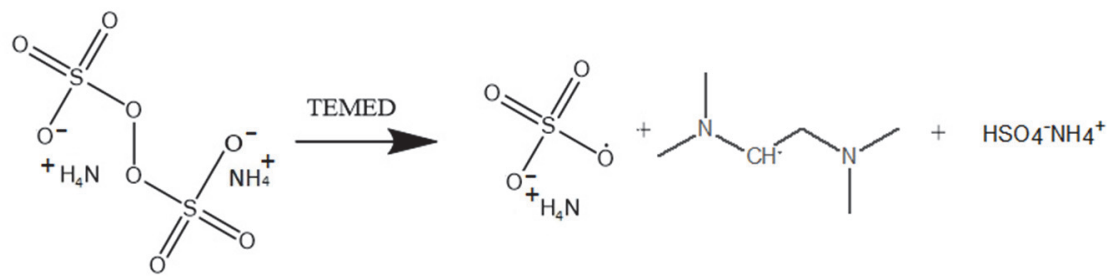<smiles>CC(C)NC(=O)CCOS(=O)(=O)[OH+]N</smiles><smiles>C=CC(=O)NC(C)C</smiles>

Fig. 3. Initiation of copolymerization scheme

a)<smiles>C=CC(=O)NC(C)C</smiles>

b)<smiles>C=CCC(C)(C)C(C)(C)C(=O)NC(C)C</smiles>

Fig. 4. Schematic representation for the synthesis of MAA-ended P1 (a) and DADMAC-ended P2

Due to the salinity of the suspension one should expect that the LCST value will be altered compared to the pure polymer solution. For that reason, appropriate solutions of polymers were prepared using flotation tailing filtrate as a base liquid and the transmittance was measured using Hitachi U-2800 A spectrophotometer at a wavelength of $500 \mathrm{~nm}$. The flocculation experiments were performed in a thermostated mixing tank equipped with 4 baffles and a mechanical marine propeller. This kind of stirrer was chosen in order to limit shear stress which would be generated e.g. by the Rushton turbine. The stirrer worked with $6.67 \mathrm{~s}^{-1}$ rotational speed. The key dimensions of the laboratory setup are presented in Fig. 5. During experiments the temperature of the thermostatic jacket as well as the temperature within the tank were monitored. Moreover a special electrode for continuous $\mathrm{pH}$ measurements of suspensions was installed. The measurement procedure was as follows. When the suspension was transferred to the mixing tank its temperature was set to $298 \mathrm{~K}$. Then, a blank sample (without the polymer) was measured with the laser sizer. Next, an appropriate dose of the polymer was injected to the suspension and the process time measurement began using a stopwatch. After that, 
samples were taken at different stages of the flocculation process. Before each PSD measurement, the background measurement was done using RO purified water. After 20 minutes of mixing the heating of suspension started. In this case also the measurements were made at different points of time as long as the steady state was achieved. In the case of both polymers the final temperature of the system was equal to $318 \mathrm{~K}$. The samples were transferred from the mixing tank to the laser sizer using a preheated plastic pipette. Moreover, the temperature of suspension within the sizer was controlled.

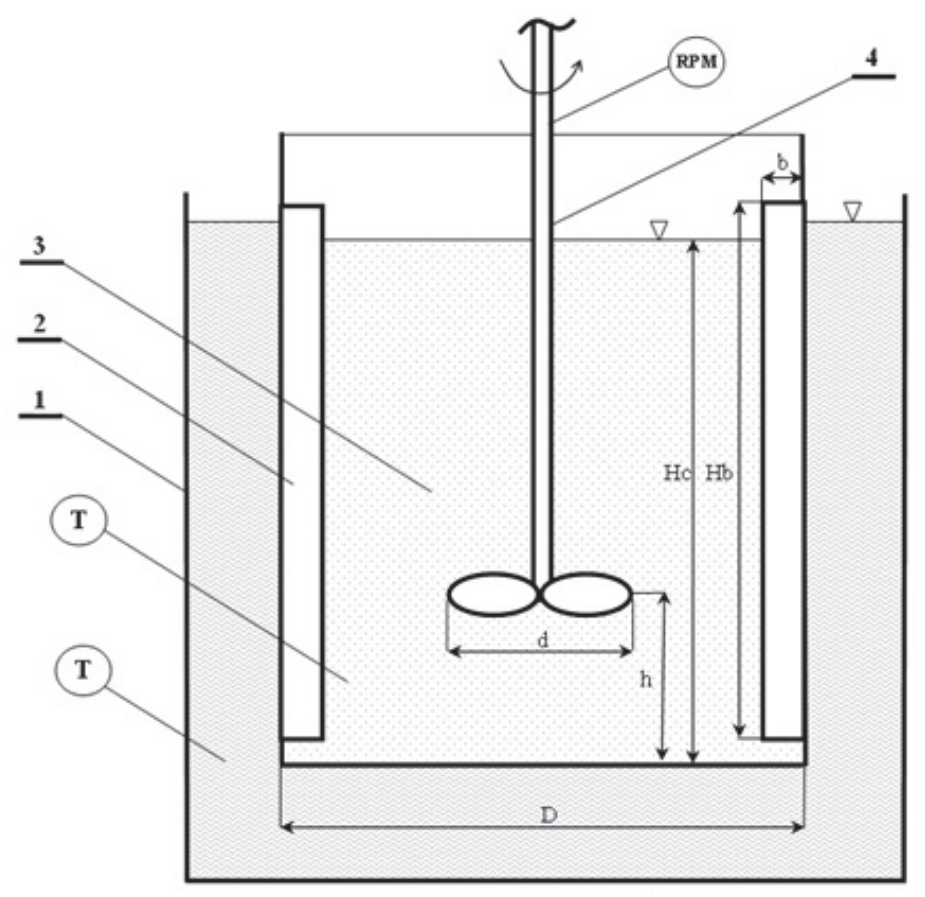

Fig. 5. Mixing tank scheme: 1) thermostatic jacket, 2) baffle, 3) mixing tank, 4) propeller. Dimensions: $D=0.098 \mathrm{~m}, d=0.038 \mathrm{~m}, b=0.01 \mathrm{~m}, H_{c}=\mathrm{D}, H_{b}=D, h=1 / 3 H_{c}$

\section{RESULTS AND DISCUSSION}

\subsection{Salinity and $\mathrm{pH}$}

Due to the salinity of samples the LCST point of polymers was determined using the filtrate solution and compared with the results for RO water solutions. The transmittance change with temperature is presented in Figs. 6 and 7. In the case of DADMAC1 sample the transition in RO water was sharp and occurred at $306 \mathrm{~K}$. This phenomenon could be observed with a naked eye because the solution became milky white. However, in the case of filtrate solution the LCST value decreased to $303 \mathrm{~K}$. Simultaneously, the sharp transition from hydrophilic to hydrophobic form was preserved. Such results should be expected as the presence of salts in the solution generally decreases the LCST (Eeckman et al., 2001). Surprisingly, in the case of P1 sample the transition behavior for the filtrate solution changed significantly. The phenomenon occurred at higher temperature compared to the RO water sample and it was more gentle. The zero transmittance was achieved above $315 \mathrm{~K}$. This fact results from the changed $\mathrm{pH}$ compared to the pure polymer solution. The $\mathrm{P} 1$ as well as $\mathrm{P} 2$ samples were $\mathrm{pH}$ sensitive because their chains possessed incorporated ionic repeating units at the end of the polymeric chain. As a result higher $\mathrm{pH}$ values increased substantially the location of the LCST point. The $\mathrm{pH}$ of suspension was equal to 7.6. This parameter was also monitored during the flocculation process. However, it did not change significantly after polymer injection also for P2 sample nor P1 sample compared to the fluctuations resulting from turbulent mixing. 


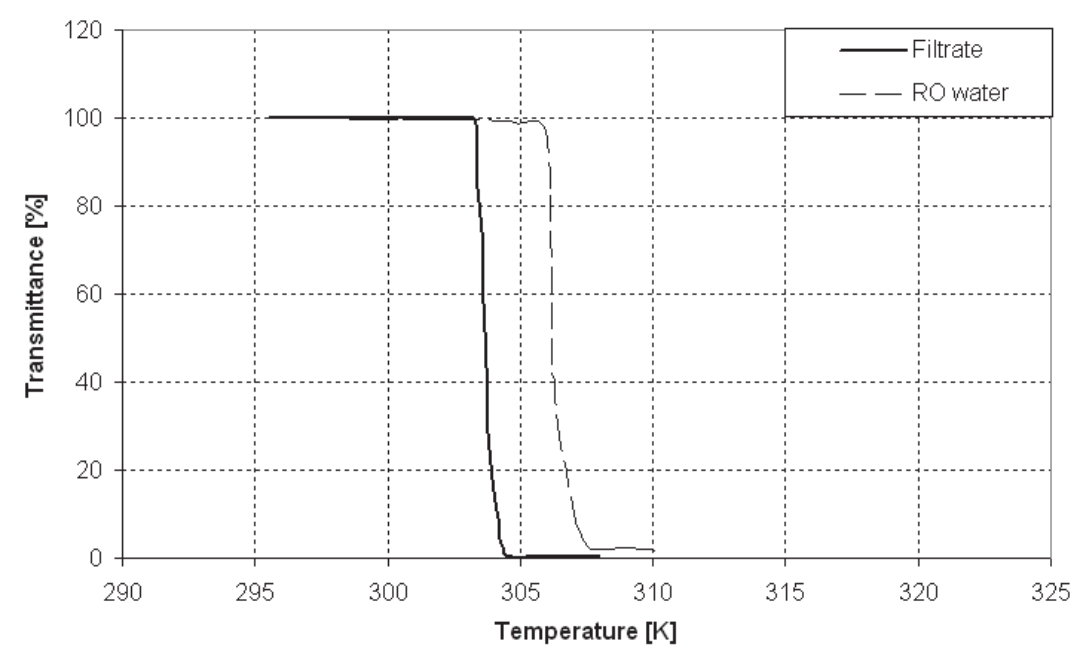

Fig. 6. The LCST determination of DADMAC1 polymer in RO water and flotation filtrate

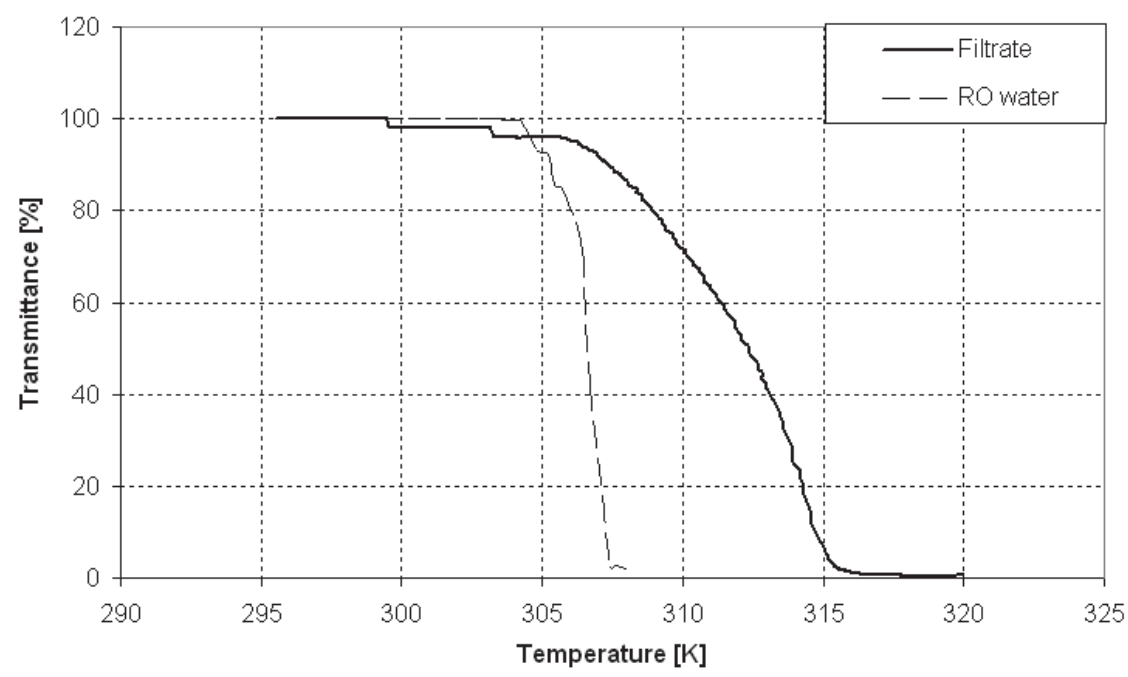

Fig. 7. The LCST determination of P1 polymer in RO water and flotation filtrate

These results reveal the first important issue of the practical application of thermosensitive polymers as flocculants, especially in the processing of minerals. The problem is that the chemical and physical properties of raw materials differ significantly, especially in the case of mineral processing. One may expect that the salinity of the feed will vary depending on acquisition site. It is probable that the change of $\mathrm{pH}$ and salt concentration will change the LCST value of a thermosentitive polymer above the range of operation of the production plant. As a result the polymer that was effective in the case of one sample may be totally ineffective for another one. On the other hand, there is a possibility that the LCST value will decrease below the feed temperature. In such a case the polymer will take the hydrophobic form immediately after its addition to the suspension, which of course, makes its application pointless. This is a significant disadvantage compared to standard flocculants used in mineral processing. In the standard process the effectiveness o flocculation can be controlled via polymer dosage. Moreover, such flocculating agents are less sensitive to the process conditions.

\subsection{Flocculation}

The flocculation process course was investigated by the analysis of particle size distribution. The data presented in Figs. 8 to 14 are summarized in the table attached as supplementary materials. P2 polymer was added to the suspension at $298 \mathrm{~K}$. Unfortunately, this flocculant proved to be efficient below LCST 
point (Fig. 8). After injection, very large aggregates were created rapidly. After 5 minutes of the process the Sauter mean diameter was equal to $9.19 \mu \mathrm{m}$ and the largest aggregates had the size of $170 \mu \mathrm{m}$. Ten minutes later, at the steady state of the process, a part of the biggest aggregates underwent breakage thus the mean diameter decreased to $8.93 \mu \mathrm{m}$. When the suspension was heated, the PSD moved insignificantly toward larger sizes (Fig. 9). The mean diameter rose to $10.85 \mu \mathrm{m}$. However, no bigger aggregates were created. It has to be emphasized that the change in the PSD was very small compared to the initial process below LCST.

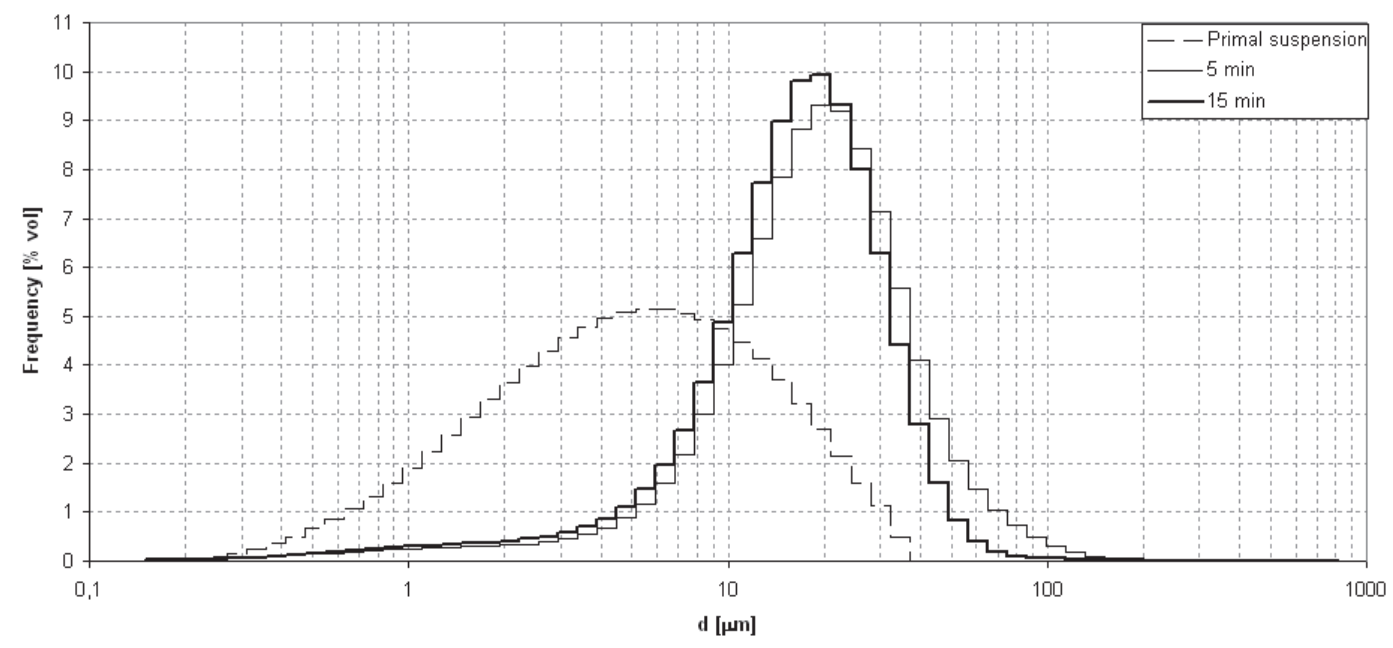

Fig. 8. PSDs for the thermosensitive flocculation using $1.0 \mathrm{mg} / \mathrm{g}$ of $\mathrm{P} 2$

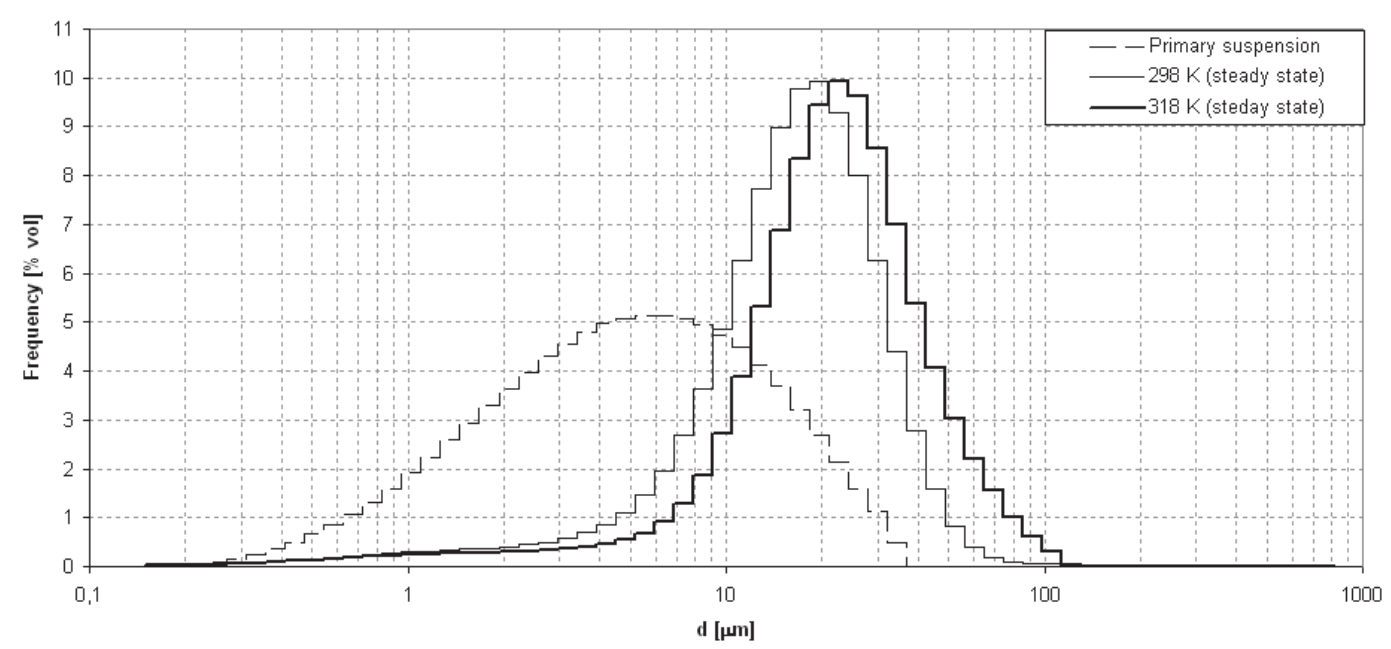

Fig. 9. PSDs for the thermosensitive flocculation using $1.0 \mathrm{mg} / \mathrm{g}$ of $\mathrm{P} 2$

When the polymer dose was decreased tenfold, i.e. it was more similar to the polymer doses used in the existing flotation plants, once again flocculation occurred (Fig. 10). However, the effect was very week. Practically, no larger aggregates were created. The PSD moved toward larger dimensions. When the suspension was heated, no change in the PSD was noticed. These facts are in agreement with the mechanism of thermosensitive flocculation. If polymer chains successfully bridge solid particles it will be very difficult for them to undergo the hydrophilic-hydrophobic transition. Therefore, at temperatures above LCST almost no aggregation effect was visible. As expected the results for P1 polymer were very similar (Figs. 11 and 12). After injection of the polymer solution a rapid growth of aggregates occurred. The change of the system was visible with a naked eye. At 5th minute of the process the largest aggregates had the size above $50 \mathrm{~m}$, whereas the Sauter mean diameter was equal to $7.74 \mu \mathrm{m}$. 


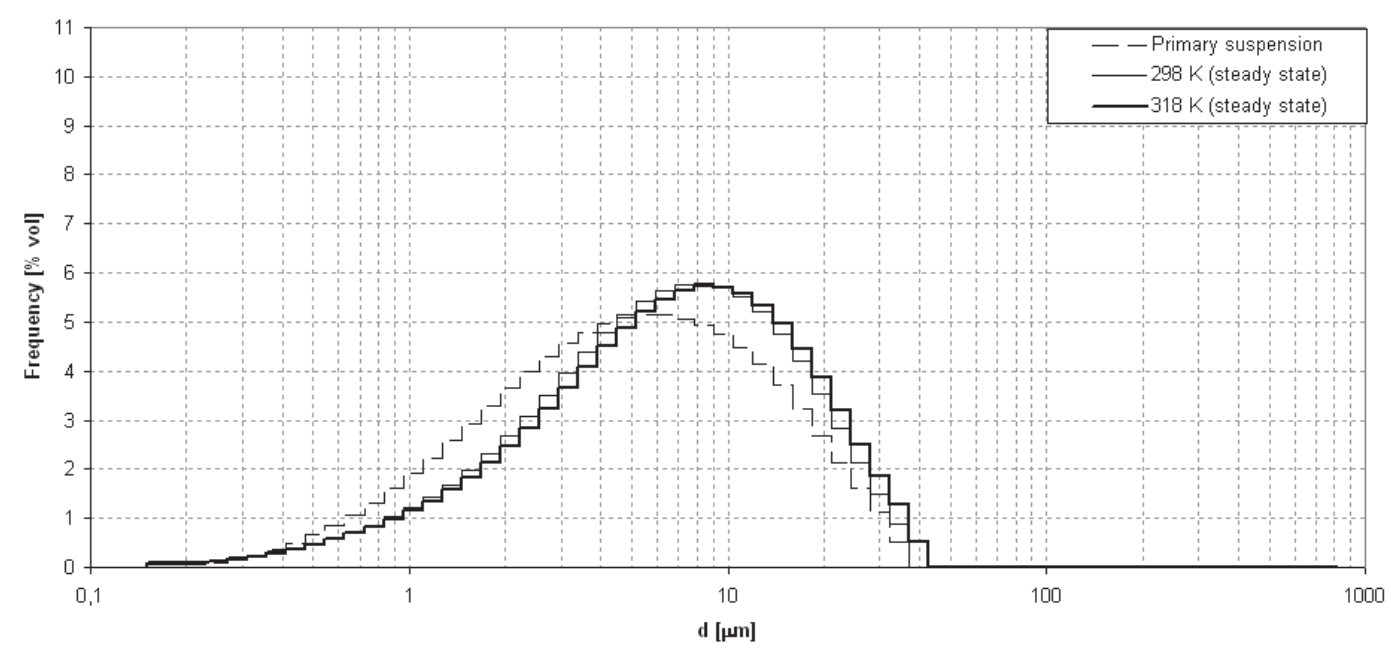

Fig. 10. PSDs for the thermosensitive flocculation using $0.1 \mathrm{mg} / \mathrm{g}$ of P2

The dominant value of particle diameter was approximately the same as in the case of DADMAC1 polymer. The lack of particles larger than $100 \mu \mathrm{m}$ and resulting smaller mean diameter can be easily explained by the electrostatic interactions between polymer ionic groups and the surface charge of particles. The zeta potential of the particles was negative, thus the macromolecule possessing cationic groups proved to be more efficient than the polymer which possessed negatively charge groups. Due to the fact that the bridging mechanism of flocculation was dominating the differences are not very large, yet they are visible.

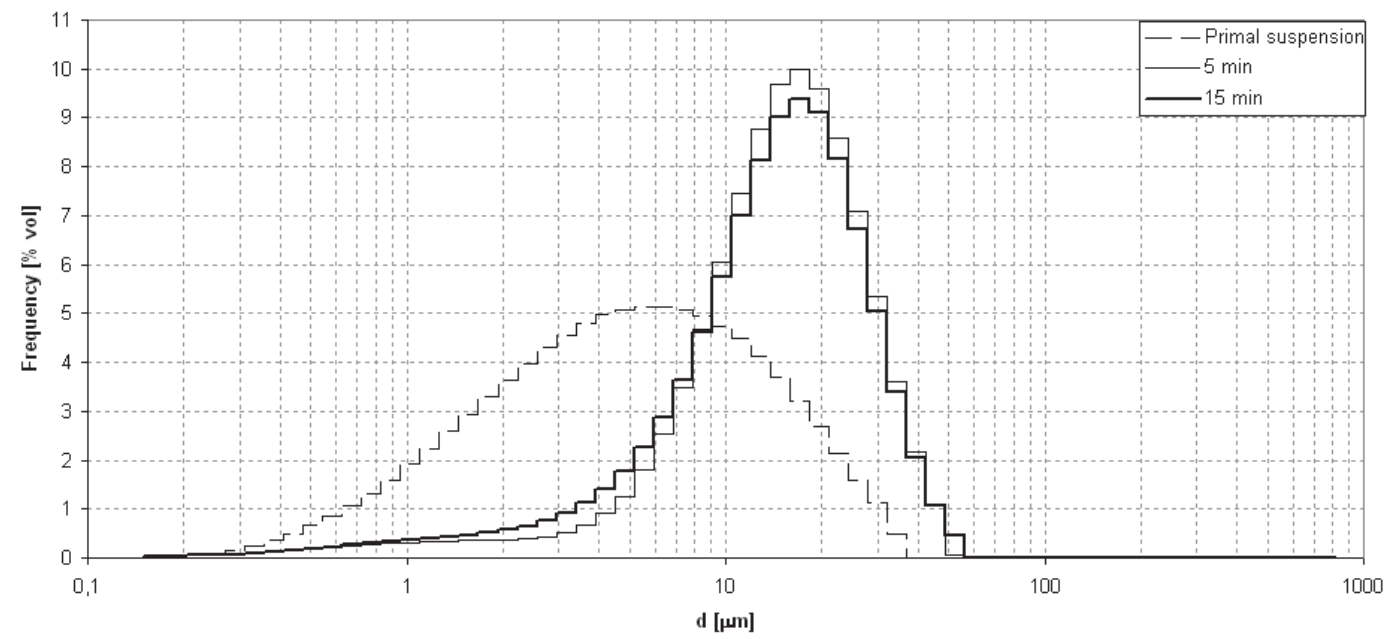

Fig. 11. PSDs for the thermosensitive flocculation using $1.0 \mathrm{mg} / \mathrm{g}$ of P1

When a smaller polymer dose was used (Fig. 13) practically no change of the PSD was noticed. Unfortunately, when the system was heated also no larger aggregates were created. Therefore, it was decided to use a high, economically unjustified overdose of P1 polymer which would guarantee the steric stabilization of solid particles and as a result strong thermosensitive behavior of the polymer. A dose equal to $10 \mathrm{mg} / \mathrm{g}$ was chosen (Fig. 14). One may notice that for the temperature below LCST significant larger aggregates were created (larger than $100 \mu \mathrm{m}$ ). This means that the range of flocculating window for this system of suspension-polymer is extremely wide. In result, one should expect that full stabilization effect will occur for significantly higher polymer dosages. On the other hand, the impact of temperature on the PSD was clearly visible in this case. Although no larger aggregates were created after heating (inhibition due to bridging) still the mean particle diameter increased from 6.38 to $15.85 \mu \mathrm{m}$. 


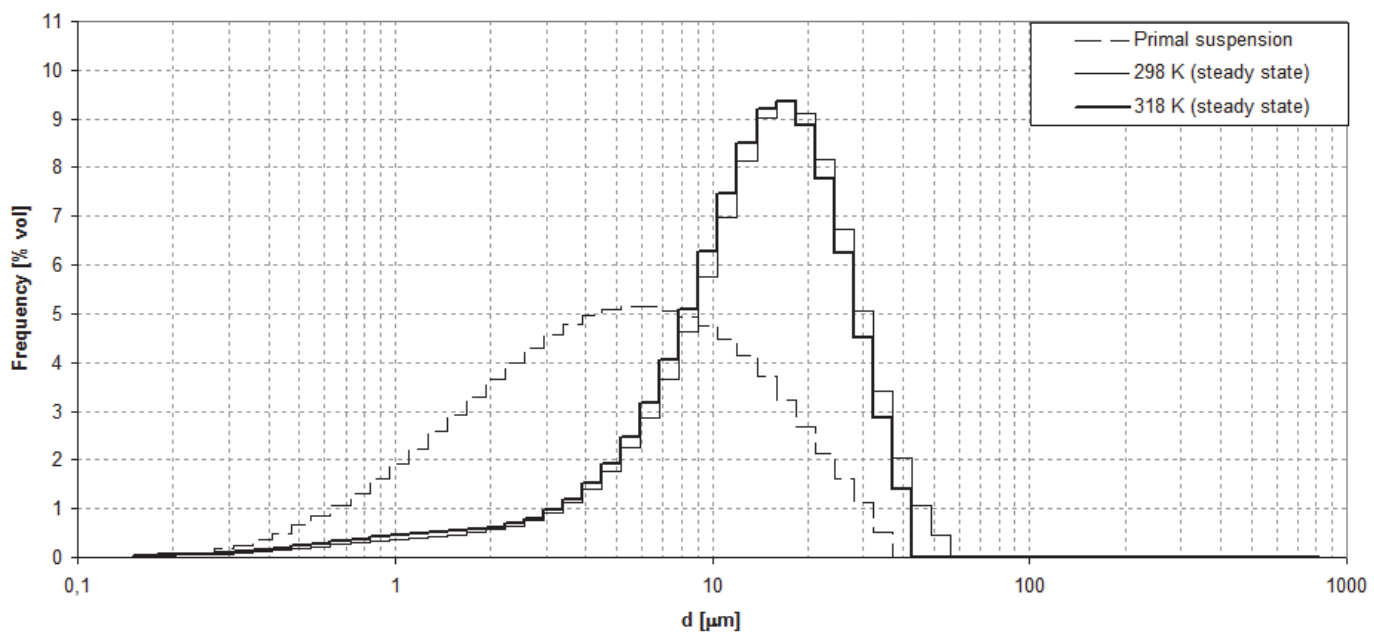

Fig. 12. PSDs for the thermosensitive flocculation using $1.0 \mathrm{mg} / \mathrm{g}$ of P1

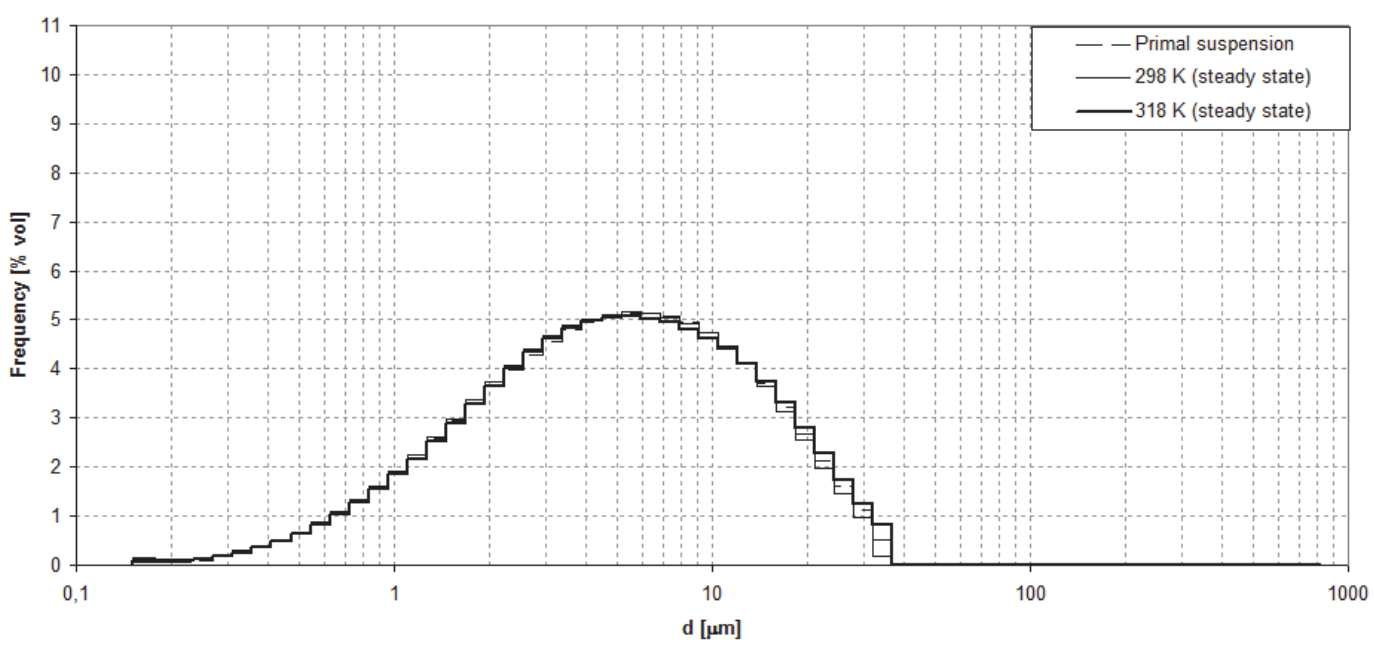

Fig. 13. PSDs for the thermosensitive flocculation using $0.1 \mathrm{mg} / \mathrm{g}$ of $\mathrm{P} 1$

In all experiments described above the molar fraction of ionic groups in the polymer chains was approximately equal to $1 \%$. In general, the reactivity of DADMAC is much lower than that of NIPAM. Although direct kinetic data for copolymerization of NIPAM/DADMAC are not available, according to the literature the reactivity ratios of DADMAC and acrylamide (AM), which exhibit similar reactivity to NIPAM, are respectively: $r_{D A D M A C}=0.06 r_{A M}=6.4, r_{D A D M A C}=0.58 r_{A M}=6.7$ (Deng and Pelton, 1995). Deng and Pelton performed copolymerization of NIPAM and DADMAC. Unfortunately, they did not determine reactivity ratios of NIPAM/DADMAC. However, from their work it can be concluded that in case of molar ratio of comonomers equal to NIPAM/DADMAC $=99: 1$, the resulting fraction of repeating units in copolymer is 99.3:0.7 mol\%.

In case of NIPAM/MAA copolymerization, reactivity ratios of $r_{N I P A M}=10.2$ and $r_{M A A}=0.01$ are reported (Xue et al., 2000). Consequently, since in both cases $r_{N I P A M} \gg 1>r_{D A D M A C / M A A}$, the compositional homogeneity up to quite a high conversion of NIPAM for those copolymers is presumed. Simultaneously DADMAC or MAA was incorporated at the end of the polymeric chain and PNIPAM enriched with DADMAC-end or PNIPAM enriched with MAA-end were obtained. 


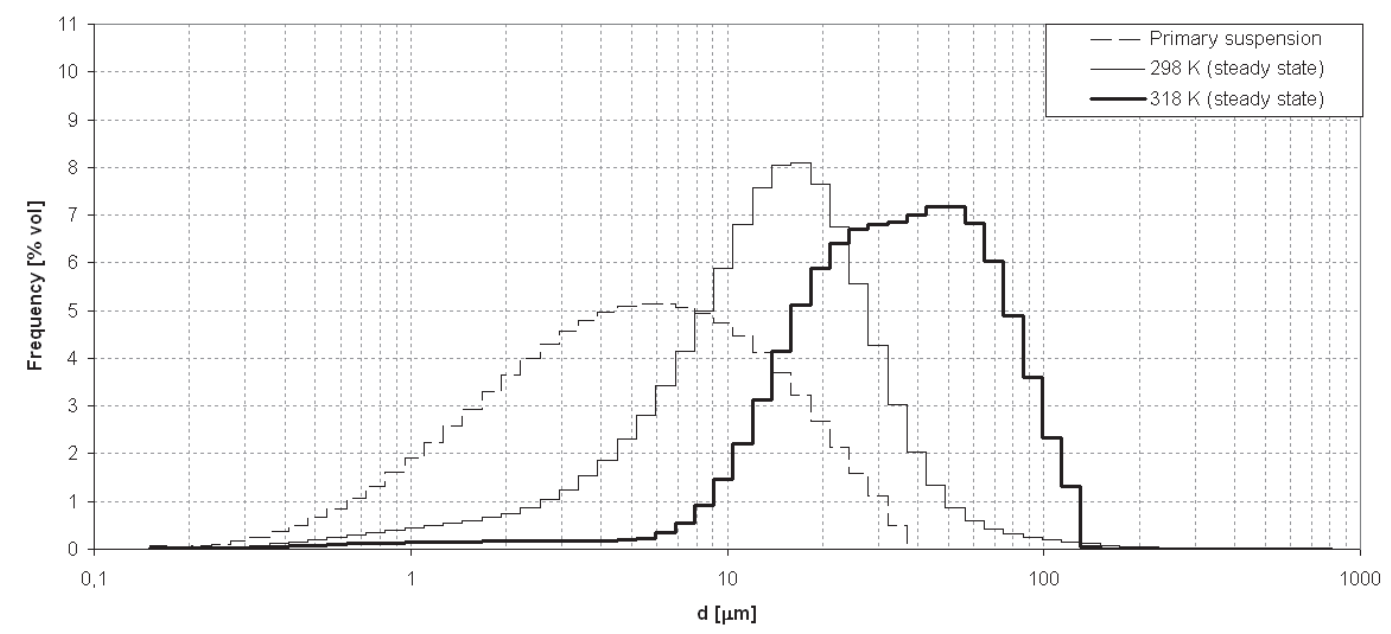

Fig. 14. PSDs for the thermosensitive flocculation using $10 \mathrm{mg} / \mathrm{g}$ of P1

Unfortunately, in the examined cases the interaction between polymers and solid particles was very strong resulting in fast flocculation via the bridging mechanism. This in turn, practically disabled thermosensitivity of the macromolecules. Of course, the structure of thermosensitive flocculants may be modified. However, one has to remember that the higher molar fraction of ionic groups, the higher the LCST point. As a result, the application of the thermosensitive polymer may be unprofitable due to high heating costs.

As was mentioned before the majority of papers concerning thermosensitive flocculation is focused on model suspensions. Therefore, it is hard to compare received experimental results. According to the authors' knowledge only the team of prof. Sakohara performed research using thermosensitive polymers in flocculation of industrial origin suspensions (Sakohara et al., 2007; Sakohara et al., 2011). As in the presented case, these authors had to overcome two substantial issues, namely the reasonable level of LCST and the amount of polymer used in the process. The incorporation of ionic groups into the polymeric chain resulted in an elevated value of transition temperature. Therefore, a method based on dual ionic thermosensitive polymers was proposed. Firstly, a cationic thermosensitive polymer was introduced to the suspension. After 5 minutes of mixing at room temperature an anionic thermosensitive polymer was added. This procedure allowed the authors to enhance dewatering process at relatively low temperatures. However, in both cases, i.e. dewatering of inorganic sludge as well as activated sludge, the dosages of polymers used in experiments were extremely high. Sakohara et al. stated that in the case of activated sludge the effective dosage was more than $3 \% \mathrm{w} / \mathrm{w}$ which is three times more than that of commercial flocculants. In the presented research the dosage of commercial flocculant varied between $0,01 \%$ to $0,02 \%$ whereas the dosage of thermosensitive polymer equal to $1 \%$ $\mathrm{w} / \mathrm{w}$ was not enough to achieve desirable results.

The last issue associated with the practical application of thermosensitive polymers in mineral processing is the economy. First of all, the cost of standard flocculants is significantly lower compared to thermosensitive polymers. Of course, this situation would change if the mass production of such macromolecules would start. Still, at the present moment such polymer substitution would be expensive. Moreover, in order to fully take advantage of thermosensitive transition steric stabilization should be achieved which in consequence results in significantly higher polymer dosages compared to standard flocculants. Combined with a higher polymer price, the application of such flocculants would be even more expensive. Finally, the application of standard flocculants does not require any additional feed processing. In turn, in the case of thermosensitive polymers a large amount of suspension should be heated above the LCST. 


\section{CONCLUSIONS}

In this paper the idea of thermosensitive polymer application in industry is discussed. Experimental results concerning flotation flocculation are presented. In experiments an industrial suspension was investigated. It was proved that there are some key issues concerning the application of thermosensitive polymers like the salinity or strong interactions between particle surface and polymer chains. Yet, thermosensitive polymers may be very efficient in some applications where the selectivity and hydrophobicity are of key importance. In the authors' opinion, thermosensitive polymers should be rather employed in processes where the reversibility of the aggregation process is fully utilized and the same polymer could be used many times in cyclic operations.

This research was supported by the Ministry of Science and Higher Education (Poland) under grant IUVENTUS PLUS no. IP2012019972.

\section{REFERENCES}

Budgin A.M., Kabachii Y.A., Shifrina Z.B., Valetsky P.M., Kochev S.S., Stein B.D., Malyutin A., Bronstein L.M., 2012. Functionalization of magnetic nanoparticles with amphiphilic block copolymers: Self-assembled thermoresponsive submicrometer particles. Langmuir, 28, 4142-4151. DOI: 10.1021/la205056k.

Burdukova E., Ishida N., Shaddick T., Franks G.V., 2011. The size of particle aggregates produced by flocculation with PNIPAM, as a function of temperature. J. Colloid. Interf. Sci., 354, 82-88. DOI: 10.1016/j.jcis.2010.10.016.

Burdukova E., Li H., Bradshaw D.J., Franks G.V., 2010a. Poly (N-isopropylacrylamide) (PNIPAM) as a flotation collector: Effect of temperature and molecular weight. Miner. Eng., 23, 921-927. DOI: 10.1016/j.mineng.2010.03.003.

Burdukova E., Li H., Ishida N., O’Shea J.P., Franks G.V., 2010b. Temperature controlled surface hydrophobicity and interaction forces induced by poly (N-isopropylacrylamide). J. Colloid. Interf. Sci., 342, 586-592. DOI: 10.1016/j.jcis.2009.10.049.

Cheng H., Shen L., Wu C., 2006. LLS and FTIR studies on the hysteresis in association and dissociation of poly(N-isopropylacrylamide) chains in water. Macromolecules, 39, 2325-2329. DOI: 10.1021/ma052561m.

Deng Y., Pelton R., 1995. Synthesis and solution properties of poly(N-isopropylacrylamide-codiallyldimethylammonium chloride). Macromolecules, 28, 4617-4621. DOI: 10.1021/ma00117a036.

Deng Y., Xiao H., Pelton R., 1996. Temperature-sensitive flocculants based on poly(N-isopropylacrylamide-codiallyldimethylammonium chloride). J. Colloid. Interf. Sci., 179, 188-193. DOI: 10.1006/jcis.1996.0201.

Eeckman F., Amighi K., Moës A.J., 2001. Effect of some physiological and non-physiological compounds on the phase transition temperature of thermoresponsive polymers intended for oral controlled-drug delivery. Int. J. Pharm., 222, 259-270. DOI: 10.1016/S0378-5173(01)00716-5.

Forbes E., 2011. Shear, selective and temperature responsive flocculation: A comparison of fine particle flotation techniques. Int. J. Miner. Process., 99, 1-10. DOI: 10.1016/j.minpro.2011.02.001.

Franks G.V., 2005. Stimulant sensitive flocculation and consolidation for improved solid/liquid separation. J. Colloid Interf. Sci., 292, 598-603. DOI: 10.1016/j.jcis.2005.06.010.

Franks G.V., Li H., O’Shea J.P., Qiao G.G., 2009. Temperature responsive polymers as multiple function reagents in mineral processing. Adv. Powder Technol., 20, 273-279. DOI: 10.1016/j.apt.2009.02.002.

Ghimici L., Constantin M., 2011. Novel thermosensitive flocculanting agent based on pullulan. J. Hazard. Mater., 192, 1009-1016. DOI: 10.1016/j.jhazmat.2011.06.002.

Gong Z., Tang D., Guo Y., 2012. The fabrication and self-flocculation effect of hybrid $\mathrm{TiO}_{2}$ nanoparticles grafted with poly(N-isopropylacrylamide) at ambient temperature via surface-initiated atom transfer radical polymerization. J. Mater. Chem., 22, 16872-16879. DOI: 10.1039/C2JM32168H.

Gregory J., Shamlou P.A. (Eds.), 1993. Processing of solid-liquid suspensions. Butterworth-Heinemann, Oxford, UK, 59-92. 
Kuźnik W., Lemanowicz M., Kuś A., Gibas M., Gierczycki A., 2010. Temperature-controlled particle size distribution of chalk suspension utilizing a thermosensitive polymer. Powder Technol., 201, 1-6. DOI: 10.1016/j.powtec.2010.02.026.

Lemanowicz M., Gierczycki A., Kuźnik W., 2016a. Review of stimuli-responsive polymers application as stabilization agents in solid-liquid dispersion systems. Polimery, 61, 92-97. DOI: 10.14314/polimery.2016.092.

Lemanowicz M., Gierczycki A., Kuźnik W., Milczyńska J., Bulanda P., 2016b. Application of thermosensitive polymers in stabilization of colloids. Adv. Powder Technol., 27, 471-480. DOI: 10.1016/j.apt.2016.01.026.

Lemanowicz M., Kuźnik W., Gibas M., Dzido G., Gierczycki A., 2012. Impact of heating method on the flocculation process using thermosensitive polymer. Water Res., 46, 4091-4098. DOI: 10.1016/j.watres.2012.05.014.

Li H., O’Shea J.P., Franks G.V., 2009. Effect of molecular weight of poly(N-isopropyl acrylamide) temperaturesensitive flocculants on dewatering. AIChE J., 55, 2070-2080. DOI: 10.1002/aic.11859.

Liu J., Yan Y., Yao P., 2011. Binding of thermo-sensitive and pH-sensitive butylated poly(allylamine)s with lysozyme. Chinese J. Polym. Sci., 29, 397-406. DOI: 10.1007/s10118-011-1054-6.

Mori T., Tsubaki J., O’Shea J.P., Franks G.V., 2013. Hydrostatic pressure measurement for evaluation of particle dispersion and flocculation in slurries containing temperature responsive polymers. Chem. Eng. Sci., 85, 38-45. DOI: 10.1016/j.ces.2012.02.014.

Qin H., Liu H., Chen Y., 2014. Influence of aliphatic amide terminals on the thermoresponsive properties of hyperbranched polyethylenimines. Chinese J. Polym. Sci., 32, 1338-1347. DOI: 10.1007/s10118-014-1509-7.

Sakohara S., Hinago R., Ueda H., 2008. Compaction of $\mathrm{TiO}_{2}$ suspension by using dual ionic thermosensitive polymers. Sep. Purif. Technol., 63, 319-323. DOI: 10.1016/j.seppur.2008.05.014.

Sakohara S., Kawachi T., Gotoh T., Lizawa T., 2013. Consolidation of suspended particles by using dual ionic thermosensitive polymers with incorporated a hydrophobic component. Sep. Purif. Technol., 106, 90-96. DOI: 10.1016/j.seppur.2012.12.030.

Sakohara S., Ochiai E., Kusaka T., 2007. Dewatering of activated sludge by thermosensitive polymers. Sep. Purif Technol., 56, 296-302. DOI: 10.1016/j.seppur.2007.02.004.

Sakohara S., Yagi S., Iizawa T., 2011. Dewatering of inorganic sludge using dual ionic thermosensitive polymers. Sep. Purif. Technol., 80, 148-154. DOI: 10.1016/j.seppur.2011.04.022.

Seidi F., Heshmati P., 2015. Synthesis of a PEG-PNIPAm Thermosensitive dendritic copolymer and investigation of its self-association. Chinese J. Polym. Sci., 33, 192-202. DOI: 10.1007/s10118-015-1561-y.

Šulc R., Lemanowicz M., Gierczycki A.T., Effect of flocculant sonication on floc growth kinetics occurring in an agitated vessel. Chem. Eng. Proc., 60, 49-54. DOI: 10.1016/j.cep.2012.05.008.

Tadros T. (Ed.), 2013. Encyclopedia of colloid and interface science. Springer-Verlag Berlin Heidelberg, New York, Dordrecht, London. DOI: 10.1007/978-3-642-20665-8.

Tokuyama H., Hisaeda J., Nii S., Sakohara S., 2010. Removal of heavy metal ions and humic acid from aqueous solutions by co-adsorption onto thermosensitive polymers. Sep. Purif. Technol., 71, 83-88. DOI: 10.1016/j.seppur.2009.11.005.

Xing X., Liu G., Ding Y., Zhang G., 2014. Revisiting the thermosensitivity of poly(acrylamide-co-diacetone acrylamide). Chinese J. Polym. Sci., 32, 531-539. DOI: 10.1007/s10118-014-1440-y.

Xue W., Champ S., Huglin M.B., 2000. Observations on some copolymerisations involving N-isopropylacrylamide. Polymer, 41, 7575-7581. DOI: 10.1016/S0032-3861(00)00171-3.

Yan J., Ji W., Chen E., Li Z., Liang D., 2010. Effect of heating rate on thermo-induced aggregation of poly(ethylene oxide)-b-poly(N-isopropylacrylamide) in aqueous solutions. Chinese J. Polym. Sci., 28, 437-447. DOI: $10.1007 / \mathrm{s} 10118-010-9085-\mathrm{y}$.

Yue G., Cui Q., Zhang Y., Wang E., Wu F., 2012. Thermo-responsive block copolymers based on linear-type poly(ethylene glycol): Tunable LCST within the physiological range. Chinese J. Polym. Sci., 30, 770-776. DOI: 10.1007/s10118-012-1179-2.

Zhang, S., Chen, C., Li, Z., 2013. Effects of molecular weight on thermal responsive property of pegylated poly-1glutamates. Chinese J. Polym. Sci., 31, 201-210. DOI: 10.1007/s10118-013-1218-7. 
APPENDIX

Table A. Volume fractions of PSDs presented in Figs. 8-14

\begin{tabular}{|c|c|c|c|c|c|c|c|c|c|c|c|c|c|c|c|}
\hline \multirow[b]{2}{*}{$\mathrm{d}, \mu \mathrm{m}$} & \multirow[b]{2}{*}{ Primal } & \multicolumn{4}{|c|}{$1.0 \mathrm{mg} / \mathrm{g}$ of $\mathrm{P} 2$} & \multicolumn{2}{|c|}{$0.1 \mathrm{mg} / \mathrm{g}$ of $\mathrm{P} 2$} & \multicolumn{4}{|c|}{$1.0 \mathrm{mg} / \mathrm{g}$ of $\mathrm{P} 1$} & \multicolumn{2}{|c|}{$0.1 \mathrm{mg} / \mathrm{g}$ of $\mathrm{P} 1$} & \multicolumn{2}{|c|}{$10 \mathrm{mg} / \mathrm{g}$ of $\mathrm{P} 1$} \\
\hline & & $5, \min$ & $15, \min$ & $298, \mathrm{~K}$ & $318, \mathrm{~K}$ & $298, \mathrm{~K}$ & $318, \mathrm{~K}$ & $5, \min$ & $15, \min$ & $298, \mathrm{~K}$ & $318, \mathrm{~K}$ & $298, \mathrm{~K}$ & $318, \mathrm{~K}$ & $298, \mathrm{~K}$ & $318, \mathrm{~K}$ \\
\hline 0.16 & 0.07 & 0.04 & 0.04 & 0.04 & 0.03 & 0.07 & 0.10 & 0.05 & 0.04 & 0.04 & 0.04 & 0.14 & 0.10 & 0.03 & 0.02 \\
\hline 0.19 & 0.05 & 0.04 & 0.04 & 0.04 & 0.04 & 0.07 & 0.10 & 0.05 & 0.04 & 0.04 & 0.05 & 0.11 & 0.07 & 0.03 & 0.02 \\
\hline 0.22 & 0.07 & 0.04 & 0.04 & 0.04 & 0.04 & 0.08 & 0.11 & 0.05 & 0.05 & 0.05 & 0.05 & 0.12 & 0.08 & 0.04 & 0.02 \\
\hline 0.25 & 0.11 & 0.05 & 0.05 & 0.05 & 0.04 & 0.11 & 0.13 & 0.06 & 0.05 & 0.05 & 0.07 & 0.16 & 0.12 & 0.05 & 0.03 \\
\hline 0.29 & 0.17 & 0.06 & 0.06 & 0.06 & 0.06 & 0.15 & 0.17 & 0.07 & 0.07 & 0.07 & 0.09 & 0.22 & 0.17 & 0.06 & 0.03 \\
\hline 0.33 & 0.25 & 0.07 & 0.08 & 0.08 & 0.07 & 0.21 & 0.22 & 0.09 & 0.09 & 0.09 & 0.12 & 0.30 & 0.25 & 0.09 & 0.04 \\
\hline 0.38 & 0.36 & 0.09 & 0.10 & 0.10 & 0.09 & 0.28 & 0.29 & 0.11 & 0.11 & 0.11 & 0.16 & 0.40 & 0.35 & 0.12 & 0.06 \\
\hline 0.44 & 0.50 & 0.11 & 0.12 & 0.12 & 0.11 & 0.36 & 0.37 & 0.14 & 0.15 & 0.15 & 0.20 & 0.53 & 0.48 & 0.15 & 0.07 \\
\hline 0.51 & 0.66 & 0.14 & 0.15 & 0.15 & 0.14 & 0.46 & 0.46 & 0.17 & 0.18 & 0.18 & 0.24 & 0.68 & 0.63 & 0.20 & 0.09 \\
\hline 0.59 & 0.85 & 0.16 & 0.19 & 0.19 & 0.16 & 0.57 & 0.57 & 0.21 & 0.22 & 0.22 & 0.29 & 0.87 & 0.81 & 0.25 & 0.10 \\
\hline 0.67 & 1.07 & 0.19 & 0.22 & 0.22 & 0.18 & 0.70 & 0.69 & 0.24 & 0.26 & 0.26 & 0.33 & 1.08 & 1.02 & 0.30 & 0.11 \\
\hline 0.78 & 1.32 & 0.21 & 0.25 & 0.25 & 0.21 & 0.85 & 0.82 & 0.27 & 0.30 & 0.30 & 0.38 & 1.33 & 1.26 & 0.35 & 0.13 \\
\hline 0.89 & 1.60 & 0.24 & 0.27 & 0.27 & 0.22 & 1.02 & 0.98 & 0.30 & 0.34 & 0.34 & 0.42 & 1.61 & 1.54 & 0.40 & 0.14 \\
\hline 1.03 & 1.91 & 0.25 & 0.30 & 0.30 & 0.24 & 1.21 & 1.15 & 0.32 & 0.37 & 0.37 & 0.45 & 1.91 & 1.84 & 0.45 & 0.14 \\
\hline 1.18 & 2.24 & 0.27 & 0.32 & 0.32 & 0.25 & 1.43 & 1.34 & 0.34 & 0.40 & 0.40 & 0.48 & 2.25 & 2.17 & 0.50 & 0.15 \\
\hline 1.36 & 2.58 & 0.28 & 0.34 & 0.34 & 0.26 & 1.69 & 1.57 & 0.35 & 0.43 & 0.43 & 0.51 & 2.61 & 2.53 & 0.54 & 0.16 \\
\hline 1.57 & 2.94 & 0.29 & 0.36 & 0.36 & 0.27 & 1.98 & 1.83 & 0.35 & 0.47 & 0.47 & 0.54 & 2.98 & 2.90 & 0.60 & 0.16 \\
\hline 1.80 & 3.30 & 0.30 & 0.38 & 0.38 & 0.28 & 2.31 & 2.13 & 0.36 & 0.51 & 0.51 & 0.57 & 3.36 & 3.28 & 0.67 & 0.16 \\
\hline 2.08 & 3.65 & 0.32 & 0.41 & 0.41 & 0.30 & 2.67 & 2.47 & 0.36 & 0.57 & 0.57 & 0.62 & 3.73 & 3.65 & 0.75 & 0.17 \\
\hline 2.39 & 3.99 & 0.35 & 0.45 & 0.45 & 0.31 & 3.08 & 2.84 & 0.38 & 0.65 & 0.65 & 0.69 & 4.08 & 4.01 & 0.87 & 0.17 \\
\hline 2.75 & 4.29 & 0.39 & 0.50 & 0.50 & 0.34 & 3.51 & 3.24 & 0.43 & 0.76 & 0.76 & 0.81 & 4.40 & 4.33 & 1.04 & 0.17 \\
\hline 3.17 & 4.57 & 0.45 & 0.58 & 0.58 & 0.37 & 3.95 & 3.67 & 0.51 & 0.92 & 0.92 & 0.97 & 4.67 & 4.61 & 1.25 & 0.17 \\
\hline 3.64 & 4.79 & 0.54 & 0.70 & 0.70 & 0.41 & 4.39 & 4.10 & 0.66 & 1.13 & 1.13 & 1.20 & 4.88 & 4.83 & 1.53 & 0.17 \\
\hline 4.19 & 4.97 & 0.68 & 0.87 & 0.87 & 0.46 & 4.79 & 4.51 & 0.90 & 1.41 & 1.41 & 1.51 & 5.03 & 4.97 & 1.87 & 0.17 \\
\hline 4.83 & 5.08 & 0.88 & 1.11 & 1.11 & 0.54 & 5.15 & 4.89 & 1.27 & 1.77 & 1.77 & 1.93 & 5.12 & 5.05 & 2.30 & 0.19 \\
\hline 5.56 & 5.14 & 1.17 & 1.46 & 1.46 & 0.68 & 5.43 & 5.21 & 1.80 & 2.25 & 2.25 & 2.47 & 5.15 & 5.07 & 2.81 & 0.23 \\
\hline 6.39 & 5.13 & 1.59 & 1.97 & 1.97 & 0.90 & 5.64 & 5.47 & 2.53 & 2.87 & 2.87 & 3.18 & 5.13 & 5.03 & 3.43 & 0.34 \\
\hline 7.36 & 5.07 & 2.18 & 2.68 & 2.68 & 1.28 & 5.76 & 5.64 & 3.49 & 3.65 & 3.65 & 4.05 & 5.06 & 4.94 & 4.15 & 0.55 \\
\hline 8.47 & 4.94 & 2.99 & 3.64 & 3.64 & 1.86 & 5.78 & 5.71 & 4.68 & 4.62 & 4.62 & 5.10 & 4.93 & 4.81 & 4.98 & 0.91 \\
\hline 9.75 & 4.74 & 4.02 & 4.86 & 4.86 & 2.72 & 5.70 & 5.69 & 6.04 & 5.76 & 5.76 & 6.27 & 4.73 & 4.63 & 5.89 & 1.46 \\
\hline 11.22 & 4.48 & 5.25 & 6.27 & 6.27 & 3.89 & 5.51 & 5.57 & 7.47 & 6.98 & 6.98 & 7.46 & 4.45 & 4.40 & 6.79 & 2.21 \\
\hline 12.91 & 4.13 & 6.59 & 7.72 & 7.72 & 5.32 & 5.20 & 5.32 & 8.77 & 8.14 & 8.14 & 8.50 & 4.09 & 4.11 & 7.56 & 3.13 \\
\hline 14.86 & 3.71 & 7.84 & 8.99 & 8.99 & 6.88 & 4.76 & 4.95 & 9.68 & 9.02 & 9.02 & 9.19 & 3.64 & 3.74 & 8.04 & 4.14 \\
\hline 17.10 & 3.22 & 8.82 & 9.79 & 9.79 & 8.35 & 4.20 & 4.46 & 9.99 & 9.39 & 9.39 & 9.34 & 3.12 & 3.31 & 8.09 & 5.10 \\
\hline 19.68 & 2.68 & 9.31 & 9.92 & 9.92 & 9.45 & 3.55 & 3.87 & 9.60 & 9.11 & 9.11 & 8.86 & 2.55 & 2.81 & 7.65 & 5.89 \\
\hline 22.65 & 2.13 & 9.19 & 9.30 & 9.30 & 9.92 & 2.84 & 3.20 & 8.57 & 8.17 & 8.17 & 7.78 & 1.98 & 2.27 & 6.76 & 6.41 \\
\hline 26.07 & 1.60 & 8.42 & 8.01 & 8.01 & 9.62 & 2.14 & 2.51 & 7.09 & 6.74 & 6.74 & 6.25 & 1.44 & 1.74 & 5.57 & 6.69 \\
\hline 30.00 & 1.12 & 7.14 & 6.27 & 6.27 & 8.55 & 1.50 & 1.85 & 5.35 & 5.05 & 5.05 & 4.52 & 0.98 & 1.25 & 4.26 & 6.80 \\
\hline 34.53 & 0.50 & 5.58 & 4.41 & 4.41 & 6.98 & 0.88 & 1.27 & 3.62 & 3.40 & 3.40 & 2.87 & 0.18 & 0.83 & 3.04 & 6.87 \\
\hline 39.74 & 0.00 & 4.10 & 2.79 & 2.79 & 5.38 & 0.00 & 0.52 & 2.16 & 2.04 & 2.04 & 1.42 & 0.00 & 0.01 & 2.04 & 7.01 \\
\hline 45.74 & 0.00 & 2.92 & 1.60 & 1.60 & 4.05 & 0.00 & 0.00 & 1.10 & 1.07 & 1.07 & 0.00 & 0.00 & 0.00 & 1.33 & 7.18 \\
\hline 52.64 & 0.00 & 2.06 & 0.84 & 0.84 & 3.02 & 0.00 & 0.00 & 0.05 & 0.46 & 0.46 & 0.00 & 0.00 & 0.00 & 0.87 & 7.18 \\
\hline 60.59 & 0.00 & 1.47 & 0.40 & 0.40 & 2.21 & 0.00 & 0.00 & 0.00 & 0.01 & 0.01 & 0.00 & 0.00 & 0.00 & 0.59 & 6.83 \\
\hline 69.73 & 0.00 & 1.04 & 0.19 & 0.19 & 1.55 & 0.00 & 0.00 & 0.00 & 0.00 & 0.00 & 0.00 & 0.00 & 0.00 & 0.42 & 6.04 \\
\hline 80.25 & 0.00 & 0.73 & 0.09 & 0.09 & 1.01 & 0.00 & 0.00 & 0.00 & 0.00 & 0.00 & 0.00 & 0.00 & 0.00 & 0.32 & 4.90 \\
\hline 92.37 & 0.00 & 0.50 & 0.06 & 0.06 & 0.60 & 0.00 & 0.00 & 0.00 & 0.00 & 0.00 & 0.00 & 0.00 & 0.00 & 0.25 & 3.59 \\
\hline 106.31 & 0.00 & 0.32 & 0.05 & 0.05 & 0.31 & 0.00 & 0.00 & 0.00 & 0.00 & 0.00 & 0.00 & 0.00 & 0.00 & 0.20 & 2.34 \\
\hline 122.35 & 0.00 & 0.19 & 0.04 & 0.04 & 0.04 & 0.00 & 0.00 & 0.00 & 0.00 & 0.00 & 0.00 & 0.00 & 0.00 & 0.16 & 1.31 \\
\hline 140.81 & 0.00 & 0.10 & 0.04 & 0.04 & 0.00 & 0.00 & 0.00 & 0.00 & 0.00 & 0.00 & 0.00 & 0.00 & 0.00 & 0.12 & 0.06 \\
\hline 162.06 & 0.00 & 0.03 & 0.04 & 0.04 & 0.00 & 0.00 & 0.00 & 0.00 & 0.00 & 0.00 & 0.00 & 0.00 & 0.00 & 0.09 & 0.00 \\
\hline 186.52 & 0.00 & 0.00 & 0.03 & 0.03 & 0.00 & 0.00 & 0.00 & 0.00 & 0.00 & 0.00 & 0.00 & 0.00 & 0.00 & 0.06 & 0.00 \\
\hline 214.66 & 0.00 & 0.00 & 0.00 & 0.00 & 0.00 & 0.00 & 0.00 & 0.00 & 0.00 & 0.00 & 0.00 & 0.00 & 0.00 & 0.04 & 0.00 \\
\hline 247.06 & 0.00 & 0.00 & 0.00 & 0.00 & 0.00 & 0.00 & 0.00 & 0.00 & 0.00 & 0.00 & 0.00 & 0.00 & 0.00 & 0.02 & 0.00 \\
\hline 284.34 & 0.00 & 0.00 & 0.00 & 0.00 & 0.00 & 0.00 & 0.00 & 0.00 & 0.00 & 0.00 & 0.00 & 0.00 & 0.00 & 0.02 & 0.00 \\
\hline 327.25 & 0.00 & 0.00 & 0.00 & 0.00 & 0.00 & 0.00 & 0.00 & 0.00 & 0.00 & 0.00 & 0.00 & 0.00 & 0.00 & 0.02 & 0.00 \\
\hline 376.64 & 0.00 & 0.00 & 0.00 & 0.00 & 0.00 & 0.00 & 0.00 & 0.00 & 0.00 & 0.00 & 0.00 & 0.00 & 0.00 & 0.00 & 0.00 \\
\hline
\end{tabular}

